\title{
Prognostic Factors Associated with Survival in Patients Infected with COVID-19: A Retrospective Study on 214 Patients from Iran
}

\author{
Amir Sadeghi, MD; Pegah Eslami, MD'; Arash Dooghaie Moghadam, MD'; Bobak Moazzami, MD²; Ali Pirsalehi, MD ${ }^{1 *}$; Saba \\ Ilkhani, MD'; Sepideh Banar , MD'; Fateme Feizollahi, MD; Mohammad Vahidi, MD'; Saeed Abdi, MD'; Hamid Asadzadeh \\ Aghdaei, MD ${ }^{3}$; Mohammad Reza Zali, MD ${ }^{*}$; Maryam Nasserinejad, MSc ${ }^{1}$ \\ ${ }^{1}$ Gastroenterology and Liver Diseases Research Center, Research Institute for Gastroenterology and Liver Diseases, Shahid Beheshti \\ University of Medical Sciences, Tehran, Iran \\ 2Department of Immunology, School of Medicine, Tehran University of Medical Sciences, Tehran, Iran \\ ${ }^{3}$ Basic and Molecular Epidemiology of Gastroenterology Disorders Research Center, Shahid Beheshti University of Medical Sciences, \\ Tehran, Iran
}

\begin{abstract}
Background: Decision-making on allocating scarce medical resources is crucial in the context of a strong health system reaction to the coronavirus disease 2019 (COVID-19) pandemic. Therefore, understanding the risk factors related to a high mortality rate can enable the physicians for a better decision-making process.

Methods: Information was collected regarding clinical, demographic, and epidemiological features of the definite COVID-19 cases. Through Cox regression and statistical analysis, the risk factors related to mortality were determined. The Kaplan-Meier curve was used to estimate survival function and measure the mean length of living time in the patients.

Results: Among about 3000 patients admitted in the Taleghani hospital as outpatients with suspicious signs and symptoms of COVID-19 in 2 months, 214 people were confirmed positive for this virus using the polymerase chain reaction (PCR) technique. Median time to death was 30 days. In this population, $24.29 \%$ of the patients died and $24.76 \%$ of them were admitted to the ICU (intensive care unit) during hospitalization. The results of Multivariate Cox regression Analysis showed that factors including age $(\mathrm{HR}, 1.031 ; 95 \% \mathrm{Cl}, 1.001-1.062 ; P$ value $=0.04)$, and C-reactive protein $(\mathrm{CRP})(\mathrm{HR}, 1.007 ; 95 \% \mathrm{Cl}, 1.000-1.015 ; P$ value $=0.04)$ could independently predict mortality. Furthermore, the results showed that age above 59 years directly increased mortality rate and decreased survival among our study population.

Conclusion: Predictor factors play an important role in decisions on public health policy-making. Our findings suggested that advanced age and CRP were independent mortality rate predictors in the admitted patients.

Keywords: COVID-19, Mortality, Prognosis, Coronavirus, SARS-CoV-2

Cite this article as: Sadeghi A, Eslami P, Dooghaie Moghadam A, Moazzami B, Pirsalehi A, Ilkhani S, et al. Prognostic factors associated with survival in patients infected with Covid-19: a retrospective study on 214 patients from iran. Arch Iran Med. 2021;24(4):333-338. doi: 10.34172/aim.2021.47
\end{abstract}

Received: May 10, 2020, Accepted: December 27, 2020, ePublished: April 1, 2021

\section{Introduction}

In December 2019, an unknown disease with pulmonary manifestations appeared in Wuhan, Hubei province, China, which gradually expanded to other countries and resulted in a new tragic pandemic. The severe acute respiratory syndrome coronavirus 2 (SARS-CoV-2), the causative agent of coronavirus disease 2019 (COVID-19), as the seventh generation of the human pathogenic family of coronaviruses, was finally considered responsible for this disease. ${ }^{1,2}$ This generation of the virus causes myalgia, fever, headache, and loss of smell or taste, in addition to respiratory problems., ${ }^{3,4}$ COVID-19 had clinical characteristics similar to other respiratory diseases; however, this virus seems to be dramatically contagious and rapidly spreads from human to human. ${ }^{5,6}$ According to 171 reports published by the World Health Organization
(WHO) in July 2020, the confirmed cases were about 11874226 people worldwide, and the mortality of the disease exceeded 545,481deaths globally. ${ }^{7}$

Although most of the patients manifest mild symptoms, COVID-19 may progress to severe disease including pulmonary edema, pneumonia, multiple organ failure, acute respiratory distress syndrome, and death. ${ }^{89}$ Thus, stratifying the patients to receive more efficient treatments and preventing final negative outcomes is of paramount significance in order to reduce the mortality rate. Up to now, many scoring systems of other infectious diseases have been evaluated in terms of predicting the prognosis of this disease. According to laboratory data, many common ratios have been previously used like neutrophil-tolymphocyte ratio (NLR) or a platelet-to-lymphocyte ratio (PLR) in the case of other diseases. 
As we know, few studies have focused on laboratory prediction values and their effects on the mortality rate. Thus, this study was conducted to analyze the prognostic values of some COVID-19 patients based on laboratory records in the Taleghani hospital to determine the effects of these parameters on final prognosis and predict survival outcomes.

\section{Materials and Methods \\ Patients and Data Collection}

This retrospective, single-center work was conducted on 214 confirmed COVID-19 patients hospitalized in the Taleghani hospital from February 22 to April 20, 2020. This hospital is a tertiary referral center in Tehran, affiliated with the Shahid Beheshti University of Medical Sciences, Tehran, Iran. SARS-CoV-2 nucleic acid reverse transcriptase-polymerase chain reaction (RT-PCR) was used to detect positive patients using samples obtained from oropharyngeal sputum or swabs. The required data were extracted from the patients' medical records and follow-up records collected by trained researchers. The first patients were recruited in our study on February 22 and the last patients in our study were followed up until May 2. The time metric was based on days. Participants' laboratory and demographic data were gathered within the first 24 hours of admission in our hospital. Follow-ups were done from admission to discharge for the patients to control the risk of in-hospital death. The dates related to discharge, death, and hospitalization stay were extracted from the medical records. Moreover, outcome was defined as death or survival for all the patients.

This project was approved by the local ethics committee of Shahid Beheshti University of Medical Sciences. This study was performed following the Declaration of Helsinki and other applicable guidelines, regulations, and laws. ${ }^{10}$

\section{Statistical Analysis}

Quantitative and categorical data were expressed as median (interquartile range [IQR]) and No. (\%), respectively. Shapiro-Wilk test and Q-Q plot were chosen for testing normal distribution of data and Levene's test was utilized for equality of variances. Welch's $t$ test was used when there was no homogeneity in variances.

A comparison was made between the groups of survivors and non-survivors using the Independent-Samples t-test; when the data were not distributed normally, the MannWhitney $U$ test was applied. For qualitative data, either the Chi-Square test or Fisher's exact test were used when appropriate. All of those who participated in the study until May 2 were followed-up to assess patients' survival, using the time-to-event technique. In this study, all the patients who survived until the end of the study or were discharged as censors were considered. The effect of all the laboratory data on overall survival was studied by Univariate and multivariable Cox proportional hazards regression models and proportional hazard assumption was examined by Schoenfeld residuals test.

The significant parameters in the univariate Cox regression model entered the multivariate Cox regression model. The following covariates were used in Cox regression: age, sex, oxygen saturation at the time of admission, lymphocyte, C-reactive protein (CRP), NLR, blood urea nitrogen (BUN), hemoglobin, and systolic and diastolic blood pressures. Hazard ratios (HRs) and 95\% confidence intervals (CIs) were reported, as well. Also, the Kaplan-Meier curve was utilized to predict the survival function and measure the mean length of living time in the patients. A Kaplan-Meier curve by age was also used to compare survival between those aged under 59 years and those aged above 59 years.

To analyze the data, a two-sided test was used and a $P$ value of less than 0.05 was considered as statistically significant. Statistical analyses in our study were conducted utilizing the $\mathrm{R}$ software, version 4.0.2.

\section{Results}

\section{Description of the Study}

A total of 3000 outpatients referred to the Taleghani hospital with suspicious manifestations of COVID-19. Among them, 245 patients with confirmed positive findings of RT-PCR for SARS-CoV-2 (PCR test) were hospitalized in the Taleghani center, between February 22 and April 20, 2020. Eventually, only the data of 214 patients were analyzed and 34 patients were missed. Table 1 presents the baseline differences in demographic and laboratory data and mortality rate between the two groups of surviving and deceased patients. The median age of the study population was equal to 59 years; and the mean age was equal to $54.69 \pm 16.92$ years among the surviving patients and equal to $68.92 \pm 12.16$ years among the deceased cases. The number of male patients (119; $55.60 \%)$ was higher than females $(95 ; 44.40 \%)$ in our study population. One hundred and twenty patients had at least one comorbidity. The most prevalent comorbidity was hypertension $(27.10 \%)$, followed by cardiovascular disease (22.90\%) and diabetes (18.69\%).

\section{Observation Time and Main Outcomes}

Median time to death was 30 days $(95 \% \mathrm{Cl}=7.27-$ 52.72), varying from 7 to 52 days. Mean date of death was day $21(95 \% \mathrm{Cl}=18.06-24.57)$, ranging from 18 to 24 days (Figure 1). Fifty-five patients $(25.70 \%)$ were admitted to the ICU. Among the ICU admitted cases, 35 patients $(63.63 \%)$ died, and 20 patients $(36.36 \%)$ were discharged from the ICU. At the time of censoring, 52 patients $(24.3 \%)$ died and 162 patients (75.7\%) survived. These patients were alive until the end of the follow-up duration or were discharged. The deceased cases were significantly older compared to the surviving group (Table 1) $(P<0.001)$. There were no differences between the 
Table 1. Baseline Characteristics and Laboratory Data of COVID-19 Patients

\begin{tabular}{|c|c|c|c|}
\hline Parameters & $\begin{array}{l}\text { Surviving } \\
(n=162)\end{array}$ & $\begin{array}{c}\text { Non-surviving } \\
(n=52)\end{array}$ & $P$ Value \\
\hline Age & $52(41-65)$ & $69(60.25-76.5)$ & $<0.001^{*}$ \\
\hline Sex & & & 0.97 \\
\hline Male (\%) & $90(55.6)$ & $29(55.8)$ & - \\
\hline Female (\%) & $72(44.4)$ & $23(44.2)$ & - \\
\hline $\mathrm{SBP}(\mathrm{mm} \mathrm{Hg})$ & $110.5(120-130)$ & $116(106.250-131.5)$ & 0.96 \\
\hline $\mathrm{DBP}(\mathrm{mm} \mathrm{Hg})$ & $79.5(70-80)$ & $70(61.25-80)$ & 0.95 \\
\hline Heart rate (beats/minute) & 85 (80-95) & $87(78.5-95.75)$ & 0.26 \\
\hline Respiratory rate (beats/minute) & $18(17-19)$ & $18(16-21.5)$ & 0.73 \\
\hline Temperature $\left({ }^{\circ} \mathrm{C}\right)$ & $37.40(37-38.2)$ & $37(37-37.8)$ & 0.41 \\
\hline $\mathrm{O}_{2}$ saturation at admission ( $\left.\%\right)$ & $92(89.25-95)$ & $91(82-94)$ & $<0.001^{*}$ \\
\hline HCT & $36.10(30.92-38.78)$ & $30.99(24.81-36.00)$ & $<0.001^{*}$ \\
\hline Hemoglobin (g/dL) & $11.71(10.11-13.01)$ & $9.51(7.05-11.51)$ & $0.013^{*}$ \\
\hline Platelet $\left(\times 10^{9}\right.$ cells/L) & $181.00(143.00-246.00)$ & $181.50(82.75-294.25)$ & 0.458 \\
\hline WBC $\left(\times 10^{9}\right.$ cells/L $)$ & $5.40(3.95-7.70)$ & $5.65(2.73-9.25)$ & 0.575 \\
\hline Neutrophil $\left(\times 10^{9}\right.$ cells/L) & $4.00(2.72-5.60)$ & $5.16(2.81-7.94)$ & $0.036^{*}$ \\
\hline Lymphocyte $\left(\times 10^{9}\right.$ cells/L) & $1.00(0.75-1.50)$ & $0.75(0.30-1.02)$ & $0.002^{*}$ \\
\hline NLR & $3.56(2.33-5.67)$ & $8.00(5.00-12.75)$ & $<0.001^{*}$ \\
\hline Platelet to lymphocyte ratio & $168.82(121.05-257.42)$ & $230.05(140.30-849.58)$ & $0.025^{*}$ \\
\hline Aspartate aminotransferase to platelet ratio & $0.21(0.11-0.30)$ & $0.28(0.12-0.74)$ & 0.047 \\
\hline Monocyte $\left(\times 10^{9}\right.$ cells/L) & $0.30(0.21-0.48)$ & $0.30(0.20-0.50)$ & 0.879 \\
\hline $\operatorname{ESR}(\mathrm{mm} / \mathrm{h})$ & $27.00(14.50-49.00)$ & $38.00(19.75-62.75)$ & 0.076 \\
\hline $\mathrm{CRP}(\mathrm{mg} / \mathrm{L})$ & $16.00(6.00-40.78)$ & $35.85(12.50-80.00)$ & $0.003^{*}$ \\
\hline $\mathrm{Na}(\mathrm{mmol} / \mathrm{L})$ & $139.00(136.00-141.00)$ & $138.00(135.00-141.00)$ & 0.286 \\
\hline $\mathrm{K}(\mathrm{mmol} / \mathrm{L})$ & $4.00(3.90-4.30)$ & $4.10(3.90-4.68)$ & 0.122 \\
\hline BUN (mg/dL) & $15.00(11.00-21.00)$ & $29.00(20.00-56.00)$ & $<0.001^{*}$ \\
\hline $\mathrm{Cr}(\mathrm{mg} / \mathrm{dL})$ & $1.00(0.80-1.20)$ & $1.30(1.00-2.25)$ & $<0.001^{*}$ \\
\hline AST (units/L) & $35.00(24.00-50.00)$ & $48.00(32.50-89.50)$ & $0.001^{*}$ \\
\hline ALT (units/L) & $34.50(22.25-51.50)$ & $35.00(17.00-62.00)$ & 0.353 \\
\hline ALK-P (units/L) & $174.00(126.00-228.00)$ & $235.00(156.75-379.75)$ & $0.002^{*}$ \\
\hline Bilirubin, total (mg/dL) & $0.70(0.50-1.00)$ & $1.00(0.65-3.00)$ & 0.076 \\
\hline Bilirubin, direct (mg/dL) & $0.80(0.50-2.10)$ & $0.90(0.60-3.28)$ & $0.037^{*}$ \\
\hline PT (s) & $13.30(12.00-14.00)$ & $13.00(12.00-14.38)$ & 0.447 \\
\hline PTT (s) & $34.00(30.00-37.00)$ & $36.00(32.50-38.50)$ & $0.015^{*}$ \\
\hline INR & $1.20(1.00-1.30)$ & $1.30(1.10-1.63)$ & $<0.001^{*}$ \\
\hline LDH (units/L) & $492.50(409.25-645.75)$ & $679.00(520.00-960.00)$ & $0.007^{*}$ \\
\hline CPK (units/L) & $87.50(58.50-153.75)$ & $101.00(53.00-203.00)$ & $0.006^{*}$ \\
\hline CKMB (IU/L) & $20.00(14.00-24.00)$ & $22.00(15.00-30.00)$ & $0.001^{*}$ \\
\hline Troponin (ng/mL) & $0.00(0.00-0.02)$ & $0.01(0.00-0.41)$ & $0.008^{*}$ \\
\hline $\mathrm{PH}$ & $7.31(7.26-7.35)$ & $7.30(7.17-7.34)$ & 0.263 \\
\hline $\mathrm{PaCO}_{2}(\mathrm{kPa})$ & $46.00(38.48-53.13)$ & $41.30(33.10-49.78)$ & 0.087 \\
\hline $\mathrm{PaO}_{2}(\mathrm{kPa})$ & $35.30(26.00-45.80)$ & $36.35(26.53-48.13)$ & 0.725 \\
\hline $\mathrm{HCO}_{3}(\mathrm{mmol} / \mathrm{L})$ & $23.65(19.18-26.92)$ & $20.70(16.15-24.83)$ & $0.016^{*}$ \\
\hline
\end{tabular}

SBP, systolic blood pressure; DBP, diastolic blood pressure; HCT, hematocrit; WBC, white blood cells; NLR, neutrophil to lymphocyte ratio; ESR, erythrocyte sedimentation rate; CRP, C-reactive protein; AST, Aspartate aminotransferase; BUN, blood urea nitrogen; Cr, creatinine; ALT, alanine aminotransferase; ALK-P, alkaline phosphatase level; PTT, Partial thromboplastin time; PT, prothrombin time; INR, international normalized ratio; LDH, Lactate dehydrogenase; CK-MB, Creatine kinase-MB; CPK, Creatine phosphokinase.

" $P$ values less than 0.05 has been regarded as significant.

surviving and deceased groups in terms of vital signs, such as respiratory rate $(P=0.73)$, heart rate $(P=0.26)$, and temperature $(P=0.41)$ while oxygen saturation was considerably lower in the deceased group (mean \pm $\mathrm{SD}=85.01 \pm 10.84)$ in comparison with the surviving group $($ mean $\pm \mathrm{SD}=90.79 \pm 5.08)(P<0.001)$. Among laboratory data on admission, hemoglobin $(P=0.01)$ and lymphocyte count $(P=0.001)$ were significantly lower in the deceased patients compared to the surviving patients. NLR $(P<0.001)$ and PLR $(P<0.001)$ were significantly different between these two groups. Mean NLR and PLR were equal to $7.72(3.34-12.35)$ and 18.65 (8.97-33.71) in the deceased patients, respectively. Mean CRP was equal to 35.85 (12.50-80.00) and 16.00 (6.00-40.78) in the deceased and surviving groups, respectively, which was significantly higher in the deceased group $(P<0.001)$. Regarding biochemistry laboratory data, creatinine $(P<0.001)$ and BUN $(P<0.001)$ were significantly higher in the deceased patients compared to the other group. In this study, variances were adjusted based on body mass index $(P$ value $=0.47)$, diabetes $(P$ value $=0.30)$, positive history of hypertension $(P$ value $=0.20)$, ischemic heart 


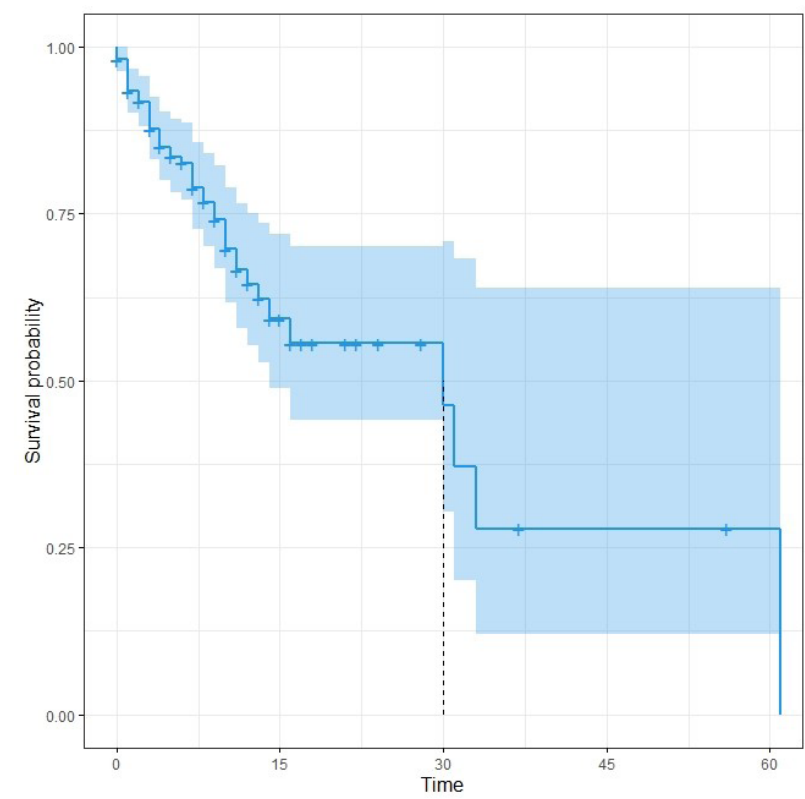

Figure 1. Kaplan-Meier Curve for Patient Survival in Admitted COVID-19 Patients $(n=214)$.

diseases $(P$ value $=0.17)$, and renal failure $(P$ value $=0.11)$.

\section{Univariate Analysis}

An increase in the age of the patients was significantly related to the mortality rate $(P<0.001 ; \mathrm{HR}, 1.053 ; 95 \%$ CI, 1.033-1.074). Survival rate was reduced in the patients older than 59 years (Figure 2). At the time of admission, a decrease in the oxygen saturation was significantly related to increased mortality rate $(P=0.004 ; \mathrm{HR}, 0.960$; 95\% CI, 0.934-0.987). A decrease in hemoglobin (HR, 0.776; 95\% CI, 0.697-0.863; $P<0.001$ ) was significantly related to mortality in our population. An increase in NLR $(\mathrm{P}<0.001 ; \mathrm{HR}, 1.076 ; 95 \% \mathrm{CI}, 1.035-1.117)$

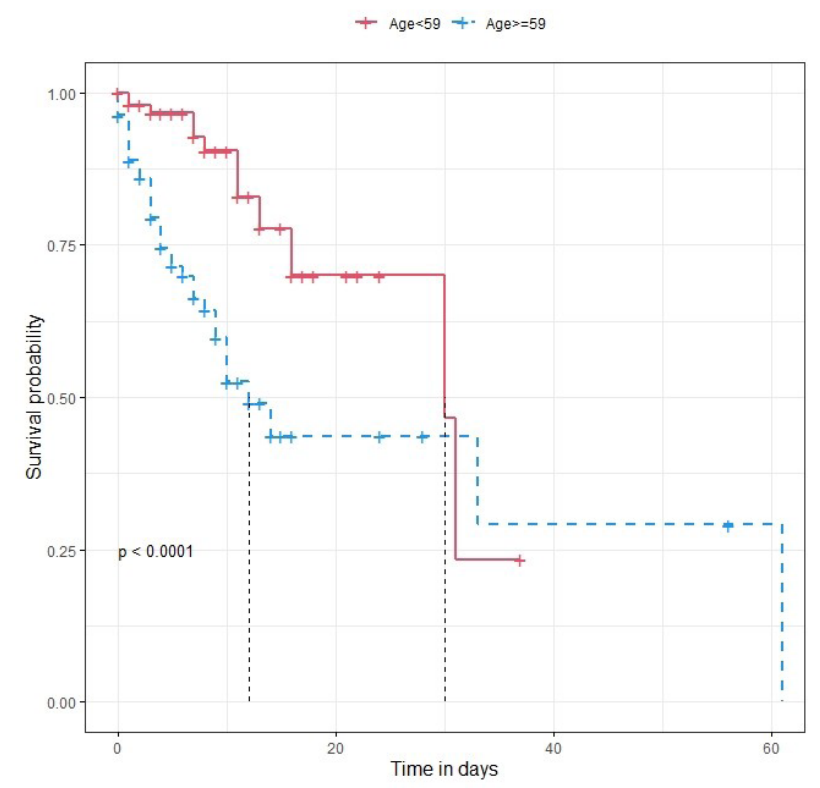

Figure 2. Survival Reported for the Whole Group and Stratified Based on Median Age ( $<59$ or $>59$ years). and a decrease in lymphocyte count (HR, 0.955; 95\% CI, $0.920-0.991 ; P=0.01$ ) could significantly reduce survival rate. Furthermore, CRP (HR, 1.008; 95\% CI, $1.004-1.013 ; P<0.001)$ and BUN (HR, 1.022; 95\% CI, $1.014-1.031 ; P<0.001)$ were significantly correlated with survival rate.

\section{Multivariate Analysis}

The results of multivariate Cox regression analysis for age parameter were as follows: $P$ value $=0.04 ; \mathrm{HR}=1.031$ and $95 \% \mathrm{CI}=1.001-1.062$. In our study population, age could be considered as an independent factor of survival in the patients infected with COVID-19. Based on the Kaplan-Meier survival chart, age above 59 years decreased survival rate (Figure 2). The analysis results of CRP were as follows: $P$ value $=0.04 ; \mathrm{HR}=1.007$; and $95 \% \mathrm{CI}=1.000$ 1.015 (Table 2). According to our results, CRP level was an independent predictor for mortality rate in the patients infected with COVID-19.

\section{Discussion}

The main objective of the present study was determining suspected laboratory items as prognostic factors among the patients admitted with confirmed COVID-19. Over the first and second weeks after the onset of symptoms, cytokines initiate an inflammatory response. ${ }^{11}$ These inflammatory reactions are associated with changes in the laboratory data and lethal risks. Therefore, the use of these predictor items in the early phases of COVID-19 infection may increase survival of the patients. Unfortunately, inflammatory pathways may not respond correctly in old patients. Therefore, old patients are asymptomatic or present with no specific manifestations. ${ }^{12,13}$ However, allocation of the diagnostic tests to the old patients, even in asymptomatic cases, could help to reduce the severity of the disease and mortality rate. ${ }^{14}$ Based on the results of the univariate Cox regression analysis, a decrease in oxygen saturation at the time of admission and hemoglobin significantly increased mortality rate. On the other hand, an increase in NLR and a decrease in lymphocyte count could significantly reduce survival rate. Furthermore, the Multivariate Cox Regression Analysis was utilized to establish a correlation between the factors including age and CRP level as independent mortality predictors in the hospitalized patients. These predictors, which are associated with survival and mortality rates, help to make optimal decisions in clinical settings. In the current study, age above 59 years decreased the survival rate significantly. Different studies have reported advanced age as a relevant indicator for predicting the severity of infection in COVID-19 patients. ${ }^{15}$ According to a study conducted in Korea, the overall mortality rate was reported at $2.36 \%$, whereas this number was increased up to $26.6 \%$ among the patients aged $\geq 80$ years. ${ }^{16}$ These results are consistent with other reports from Europe and 
Table 2. Univariate and Multivariable Cox Regression Analysis and Cox with the Time Dependent Results for Identifying Mortality-associated Risk Factors NLR, neutrophil to lymphocyte ratio; CRP, C-reactive protein; BUN, blood urea nitrogen; SBP, systolic blood pressure; DBP, diastolic blood pressure; $\mathrm{Cl}$, confidence interval; $\mathrm{HR}$, hazard ratio

\begin{tabular}{|c|c|c|c|c|c|c|}
\hline \multirow{2}{*}{ Variables } & \multicolumn{3}{|c|}{ Univariate Analysis } & \multicolumn{3}{|c|}{ Multivariate Analysis } \\
\hline & HR & $95 \% \mathrm{Cl}$ & $\boldsymbol{P}^{\mathrm{a}}$ & HR & $95 \% \mathrm{Cl}$ & $P$ \\
\hline Age & 1.053 & $1.033-1.074$ & $<0.001$ & 1.031 & $1.001-1.062$ & 0.04 \\
\hline Sex & 0.919 & $0.527-1.601$ & 0.765 & - & - & - \\
\hline Admission $\mathrm{O}_{2}$ saturation & 0.960 & $0.934-0.987$ & 0.004 & 0.985 & $0.953-1.019$ & 0.39 \\
\hline Lymphocyte count & 0.955 & $0.920-0.991$ & 0.015 & 0.943 & $0.667-1.332$ & 0.74 \\
\hline NLR & 1.076 & $1.035-1.117$ & $<0.001$ & 1.004 & $0.986-1.022$ & 0.64 \\
\hline CRP & 1.008 & $1.004-1.013$ & $<0.001$ & 1.007 & $1.000-1.015$ & 0.04 \\
\hline BUN & 1.022 & $1.014-1.031$ & $<0.001$ & 1.014 & $0.995-1.034$ & 0.13 \\
\hline Hemoglobin & 0.776 & $0.697-0.863$ & $<0.001$ & 0.871 & $0.693-1.094$ & 0.23 \\
\hline SBP & 0.997 & $0.987-1.006$ & 0.494 & - & - & - \\
\hline DBP & 0.992 & $0.973-1.011$ & 0.389 & - & - & - \\
\hline
\end{tabular}

a $P$ values less than 0.05 are significant.

the USA. For instance, in Italy, the mortality rate among old patients was determined to be four times higher than other patients. ${ }^{17}$ Furthermore, our results showed CRP as another predictor of mortality in the patients infected with COVID-19. CRP is categorized as a sensitive acute phase reactant. This factor is produced by the hepatic cells and can be increased in cardiovascular diseases, infections, and inflammatory conditions. ${ }^{18}$ Contrary to our results, in a meta-analysis conducted by Huang et al, although high levels of CRP increased the need for ICU care, the role of CRP as a survival predictor remained controversial. ${ }^{19}$ According to recent studies, unfortunately, there is not a definite cut-off point for CRP level that can exactly predict mortality in critical COVID-19 patients. ${ }^{20,21}$ On the other hand, due to the low specificity of this factor, we could not employ CRP as a diagnostic factor. In a cross-sectional study, Gao et al observed that CRP level was higher in the group with advanced age. ${ }^{22}$ So, it seems that CRP could predict the severity of the disease and mortality in the group with advanced age infected with COVID-19. ${ }^{22}$ Although CRP is not considered as a diagnostic factor due to its low specificity, it can be effective as a practical factor in monitoring the progression of the disease in hospital settings. Accordingly, these two predictors, advanced age and CRP level, can significantly predict the survival rate in critically ill patients. However, there were various limitations in the current study including small sample size and limited follow-up duration. In addition, we only had access to the patients of a single COVID-19 center. Thus, further prospective studies are required to conduct more comprehensive analysis.

In conclusion, the results of this study suggested that biomarkers, such as CRP, can change survival prognosis among critically ill patients. Moreover, the risk of mortality was significantly increased in the old patients ( $\geq 59$ years). Therefore, these survival predictors can be utilized as an auxiliary approach in clinical practice to guide treatment in critically ill patients. However, to confirm these results, larger sample sizes and more studies are required.

\section{Authors' Contribution}

AS, MRZ and AP: Conceived of the presented idea. AS, PE, ADM, SA and HAA: Conceived and designed the analysis. AP, SI, SB, FF and MV: Collected data. BM, MN, AP and SI: Contributed data or analysis tools. BM, MN, SB, FF and MV: Perform the analysis. PE and ADM: Wrote the paper. MRZ, AP and HAA: Involved in planning and supervised the work. All authors discussed the results and commented on the manuscript.

\section{Conflict of Interest Disclosures}

All authors declare no conflicts of interests.

\section{Ethical Statement}

The study was approved by the Ethics Committee of Shahid Beheshti Medical University.

\section{References}

1. Prevention cfdca. Human Coronavirus Types 2020. Available from: https://www.cdc.gov/coronavirus/types.html.

2. Moazzami B, Razavi-Khorasani N, Dooghaie Moghadam A, Farokhi E, Rezaei N. COVID-19 and telemedicine: Immediate action required for maintaining healthcare providers wellbeing. J Clin Virol. 2020;126:104345. doi: 10.1016/j. jcv.2020.104345.

3. Li LQ, Huang T, Wang YQ, Wang ZP, Liang Y, Huang TB, et al. COVID-19 patients' clinical characteristics, discharge rate, and fatality rate of meta-analysis. J Med Virol. 2020;92(6):577583. doi: 10.1002/jmv.25757.

4. Gautier JF, Ravussin Y. A new symptom of COVID-19: loss of taste and smell. Obesity. 2020;28(5):848. doi: 10.1002/ oby.22809.

5. Rothe C, Schunk M, Sothmann P, Bretzel G, Froeschl G, Wallrauch C, et al. Transmission of 2019-nCoV Infection from an Asymptomatic Contact in Germany. N Engl J Med. 2020;382(10):970-971. doi: 10.1056/NEJMc2001468.

6. Sadeghi A, Dooghaie Moghadam A, Eslami P, Pirsalehi A, Salari S, Roshandel E. Vasculopathy-related cutaneous lesions and intrahepatic cholestasis as a synchronous manifestations in COVID-19 patient: case report. Gastroenterol Hepatol Bed Bench. 2020;13(4):400-404.

7. World Health Organization. Coronavirus disease (COVID-19)!: situation report, 171. 2020. Available from: https://apps.who. int/iris/handle/10665/333151.

8. Chen N, Zhou M, Dong X, Qu J, Gong F, Han Y, et al. 
Epidemiological and clinical characteristics of 99 cases of 2019 novel coronavirus pneumonia in Wuhan, China: a descriptive study. Lancet. 2020;395(10223):507-513. doi: 10.1016/S0140-6736(20)30211-7.

9. Torkian P, Ramezani N, Kiani P, Bax MR, Akhlaghpoor S. Common CT Findings of Novel Coronavirus Disease 2019 (COVID-19): A Case Series. Cureus. 2020;12(3):e7434. doi: 10.7759/cureus.7434.

10. World Medical Association. World Medical Association Declaration of Helsinki: ethical principles for medical research involving human subjects. JAMA. 2013;310(20):2191-4. doi: 10.1001/jama.2013.281053.

11. Terpos E, Ntanasis-Stathopoulos I, Elalamy I, Kastritis E, Sergentanis TN, Politou M, et al. Hematological findings and complications of COVID-19. Am J Hematol. 2020;95(7):834847. doi: 10.1002/ajh.25829.

12. Castle SC. Clinical relevance of age-related immune dysfunction. Clin Infect Dis. 2000;31(2):578-85. doi: $10.1086 / 313947$.

13. Tasa D, Eslami P, Dashti H, Nassiri Toosi M, Zarghami SY, Zarghami SY, et al. The successful management of Thirtysix hepatopancreatobiliary surgeries under the intensive protective arrangements during the COVID-19 pandemic. Acta Biomed. 2020;91(3):e2020005. doi: 10.23750/abm. v91i3.9997.

14. Alsuliman $T$, Sulaiman $R$, Ismail $S$, Srour $M$, Alrstom A. COVID-19 paraclinical diagnostic tools: Updates and future trends. Curr Res Transl Med. 2020;68(3):83-91. doi: 10.1016/j. retram.2020.06.001

15. Zhang F, Wei K, Slowikowski K, Fonseka C, Rao D, Kelly S, et al. Defining inflammatory cell states in rheumatoid arthritis joint synovial tissues by integrating single-cell transcriptomics and mass cytometry. Nat Immunol. 2019;20(7):928-942. doi: 10.1038/s41590-019-0378-1.

16. Coronavirus Disease-19, Republic of Korea. Available from: http://ncov.mohw.go.kr/en/.

17. Sanità. ISd. Epidemia COVID-19. 2020. Available from: https://www.epicentro.iss.it/coronavirus/bollettino/Bollettinosorveglianza-integrata-COVID-19_2-aprile-2020.pdf.

18. Jain S, Gautam V, Naseem S. Acute-phase proteins: As diagnostic tool. J Pharm Bioallied Sci. 2011;3(1):118-27. doi: 10.4103/0975-7406.76489.

19. Huang I, Pranata R, Lim MA, Oehadian A, Alisjahbana B. C-reactive protein, procalcitonin, D-dimer, and ferritin in severe coronavirus disease-2019: a meta-analysis. Ther Adv Respir Dis. 2020;14:1753466620937175. doi: $10.1177 / 1753466620937175$.

20. Ren HW, Wu Y, Dong JH, An WM, Yan T, Liu Y, et al. Analysis of clinical features and imaging signs of COVID-19 with the assistance of artificial intelligence. Eur Rev Med Pharmacol Sci. 2020;24(15):8210-8218. doi: 10.26355/ eurrev_202008_22510.

21. Eslami P, Tabary M, Dooghaie-Moghadam A, Khaheshi I. How to overcome cardiovascular challenges in COVID-19 patients: a guide for common practice. Acta Biomed. 2020;91(3):e2020021. doi: 10.23750/abm.v91i3.9848.

22. Gao S, Jiang F, Jin W, Shi Y, Yang L, Xia Y, et al. Risk factors influencing the prognosis of elderly patients infected with COVID-19: a clinical retrospective study in Wuhan, China. Aging. 2020;12(13):12504-12516. doi: 10.18632/ aging. 103631 